\title{
CADERNOS de Ética e Filosofia Política
}

NÚMERO 31

FFLCH TCS 


\section{- CADERNOS de Ética e Filosofia Política}

\section{CADERNOS DE ÉTICA E FILOSOFIA POLÍTICA}

No 31 - São Paulo - $2^{\circ}$ semestre de 2017

Publicação semestral - ISSN 1517-0128

Editores: Maria das Graças de Souza (responsável institucional), Alessandra Tsuji, Caio Cunha Leitão, Fran de Oliveira Alavina, Mariana de Mattos Rubiano, Sandra Pires de Toledo Pedroso, Silvio Ricardo Gomes Carneiro, Taynam Santos Luz Bueno, Thiago Dias da Silva, Thiago Vargas Escobar Azevedo, Victor Fiori.

Conselho Editorial: Conselho Editorial: Alberto Ribeiro de Barros (USP), Antônio Carlos dos Santos (UFS), Cláudio Boeira Garcia (Unijuî), Jaimir Conte (UFRN), José Oscar de Almeida Marques (Unicamp), Jose Thomaz Almeida Brum Duarte (PUC-RJ), Helena Esser dos Reis (UFG), Luiz Felipe Netto de Andrade e Silva Sahd (UFU), Luiz Fernando Batista Franklin de Mattos (USP), Maria Lúcia Mello e Oliveira Cacciola (USP), Marilena de Souza Chaui (USP), Milton Meira do Nascimento (USP), Miroslav Milovic (UnB), Newton Bignotto (UFMG), Patrícia Fontoura Aranovich (Unifesp), Paulo Jonas de Lima Piva (USJT), Renato Janine Ribeiro (USP), Ricardo Monteagudo (Unesp), Rodrigo Brandão (UFPR), Rolf Kuntz (USP), Ruy Fausto (USP), Sérgio Cardoso (USP).

Imagem de capa: Paul Klee, Revolution of the Viaduct, 1937. Hamburger Kunsthalle.

\author{
USP \\ Universidade de São Paulo \\ Reitor: Prof. Dr. Marco Antônio Zago \\ Vice-reitor: Prof. Dr. Vahan Agopyan
}

FFLCH

Faculdade de Filosofia, Letras e Ciências Humanas

Diretora: Prof. Dr. Maria Arminda do Nascimento

Arruda

Vice-Diretor: Paulo Martins

Departamento de Filosofia

Chefe: Prof. Dr. Luiz Sérgio Repa

Vice-chefe: Prof. Dr. Oliver Tolle

Coordenador do Programa de Pós-Graduação:

Prof. Dr. Moacyr Ayres Novaes Filho

Prof. Dr. Marcus Sacrini Ayres Ferraz

Endereço para correspondência e aquisição:

Profa. Dra. Maria das Graças de Souza

Departamento de Filosofia - USP

Av. Prof. Luciano Gualberto, 315

05508-900 - São Paulo - SP - Brasil

Tel.: (0xx11) 3091-3761 - Fax: (0xx11) 3031-2431

E-mail: cefp@usp.br

Cadernos de Ética e Filosofia Política é uma publicação do Programa de Pós-Graduação do Departamento de

Filosofia da Universidade de São Paulo.

Os artigos deste periódico são indexados por: The Philosopher's Index e Latin Americanist Research Resources Project - LARRP

WWW.REVISTAS.USP.BR/CEFP

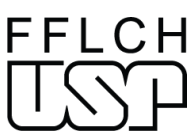

